\title{
Advanced Synthetic Aperture Radar Based on Digital Beamforming and Waveform Diversity
}

\author{
Gerhard Krieger, Nicolas Gebert, Marwan Younis, Alberto Moreira \\ Microwaves and Radar Institute, German Aerospace Center \\ 82234 Oberpfaffenhofen, Germany, email: gerhard.krieger@dlr.de
}

\begin{abstract}
This paper introduces innovative SAR system concepts for the acquisition of high resolution radar images with wide swath coverage from spaceborne platforms. The new concepts rely on the combination of advanced multi-channel SAR front-end architectures with novel operational modes. The architectures differ regarding their implementation complexity and it is shown that even a low number of channels is already well suited to significantly improve the imaging performance and to overcome fundamental limitations inherent to classical SAR systems. The more advanced concepts employ a multidimensional encoding of the transmitted waveforms to further improve the performance and to enable a new class of hybrid SAR imaging modes that are well suited to satisfy hitherto incompatible user requirements for frequent monitoring and detailed mapping. Implementation specific issues will be discussed and examples demonstrate the potential of the new techniques for different remote sensing applications.
\end{abstract}

Index Terms - Synthetic Aperture Radar (SAR), Digital Beamforming, MIMO Radar, Waveform Diversity, HighResolution Wide-Swath (HRWS) SAR Imaging.

\section{INTRODUCTION}

Wide swath coverage and high azimuth resolution pose contradicting requirements on the design of spaceborne synthetic aperture radar (SAR) systems [1]. This motivated the development of advanced SAR imaging modes with different trade-offs between spatial coverage and azimuth resolution. Examples are the ScanSAR mode which enables a wide swath at the cost of an impaired azimuth resolution [2][3] and the Spotlight mode which allows for an improved azimuth resolution at the cost of a noncontiguous imaging along the satellite track [4]. It is, however, up to now not possible to combine both operational modes simultaneously in one and the same data take. This dilemma motivated further research towards the development of new radar techniques for spaceborne high-resolution wide-swath SAR imaging.

A promising candidate for such a new radar imaging technique is digital beamforming on receive where the receiving antenna is split into multiple sub-apertures (cf. Figure 1). In contrast to analog beamforming, the received signals from each sub-aperture element are separately amplified, down-converted, and digitized. This enables an a posteriori combination of the recorded sub-aperture signals to form multiple beams with adaptive shapes. The additional information about the direction of the scattered radar echoes can then be used to (1) suppress spatially ambiguous signal returns from the ground, (2) to increase the receiving antenna gain without a reduction of the imaged area, (3) to suppress spatially localized interferences, and (4) to gain additional information about the dynamic behavior of the scatterers and their surroundings. By this, it becomes possible to overcome fundamental limitations of conventional SAR systems [5]-[17].

\section{Multi-Channel SAR Systems}

Several proposals resolve the azimuth resolution vs. coverage dilemma by combining a multi-channel radar receiver with a fixed small aperture transmitter illuminating a wide area on the ground. An early example is a multiple beam SAR operating in a squinted imaging geometry [6][7]. The squinted geometry allows for the simultaneous imaging of multiple swaths at an almost constant incident angle and the combination of the subswaths yields a wide image swath without ambiguities (Figure 2 , left). Major drawback of this system is the high squint angle that complicates the processing and impairs the performance. Another promising approach is the displaced phase centre antenna technique [5]. The basic idea behind this system is to use multiple apertures in the along-track direction and to acquire for each transmitted pulse additional samples along the synthetic aperture (Figure 2, second column). As a result, one may then reduce the transmit PRF which enables in turn the unambiguous mapping of a wider image swath. An extension of the DPCA technique is the Quad Array system [9] which employs additional apertures in elevation to suppress range ambiguous returns [8]. By this, one may further increase the image swath, but the drawback is a range gap in the middle of the wide swath since it is impossible to simultaneously transmit and receive radar pulses (Figure 2, third column). A further extension of the DPCA technique is the High Resolution Wide Swath (HRWS) SAR system [10][12]. This system combines a separate small transmit antenna with a large receiver array as illustrated in the fourth column of Figure 2. The small transmit antenna illuminates a wide swath on the ground and the large

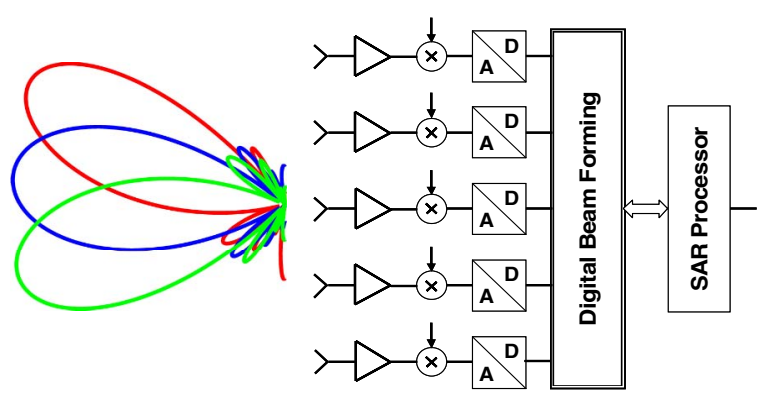

Figure 1 - Schematic of a multi-channel receiver. The signal from each subaperture element is independently amplified, down-converted, and digitized. The digital processing enables flexible and adaptive beamforming a posteriori to signal reception. 


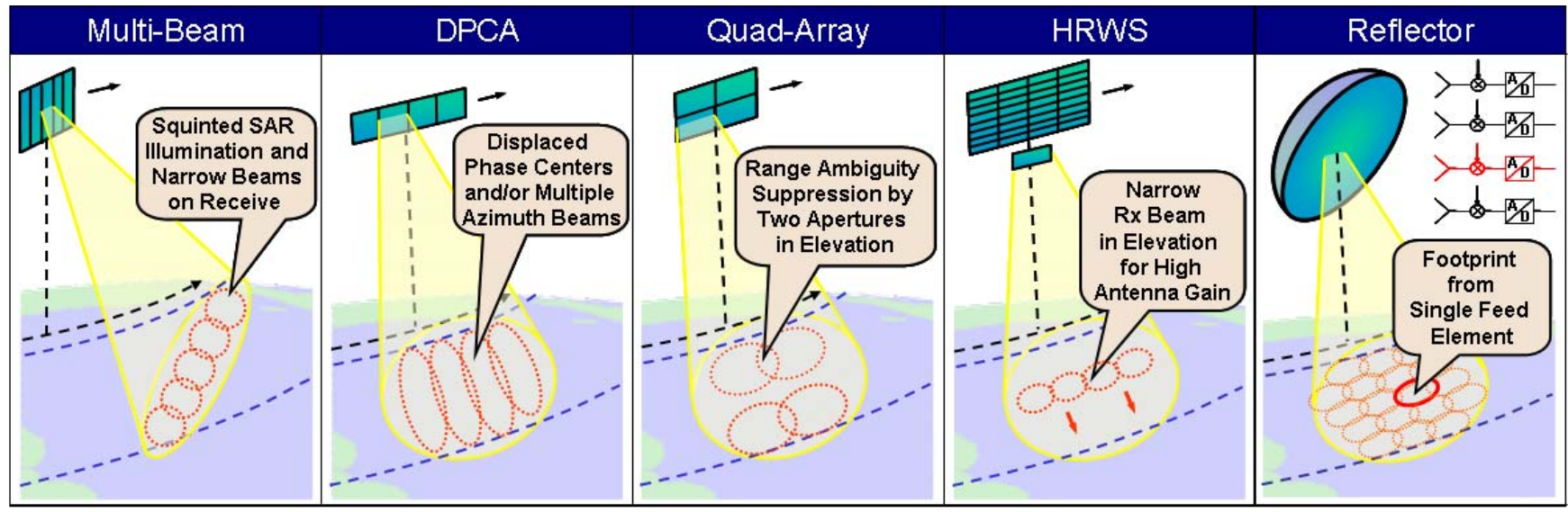

Figure 2 - Solutions for high-resolution wide-swath SAR imaging. Left: multiple beam SAR [6][7]. Middle left: displaced phase center antenna technique [5]. Middle: quad-element rectangular array SAR [9]. Middle right: high-resolution wide-swath (HRWS) SAR [10][12]. Right: High resolution wide swath SAR employing a reflector antenna in combination with a digital feed array.

receiver array compensates the Tx gain loss by a real time digital beamforming process in elevation called scanning on receive (SCORE). Multiple azimuth channels allow furthermore for the imaging of a wide swath without rising azimuth ambiguities. The combination of the azimuth signals from the multiple apertures requires the application of dedicated multichannel SAR signal processing algorithms as introduced in [11] and further elaborated in [13].

The HRWS concept relies on a fixed wide-area illumination by a separate transmit antenna. This enables an independent electrical design and optimization of the transmit and receive paths, but it requires also the accommodation of an additional antenna on the spacecraft and reduces the flexibility to operate the radar system in different SAR imaging modes like ultrawide-swath ScanSAR, high SNR spotlight, or new hybrid modes to be discussed later. It is hence worth to consider also the application of digital beamforming techniques in radar systems that use the same antenna for both the transmission and reception of radar pulses, thereby taking advantage of already existing space-qualified T/R module technology. Since a large aperture corresponds to a narrow beam, this poses in turn the question of how to distribute the signal energy on the ground. The trivial solution for a large-area direct radiating array would be amplitude tapering, or as an extreme case, the use of only a part of the antenna as suggested for the Multi-Beam or QuadArray SAR, but this causes a significant loss of efficiency. Another possible solution is phase tapering, but the derivation of appropriate phase coefficients is an intricate task which requires in general complicated numerical optimization techniques.

\section{Digital Beamforming With Reflector Antennas}

An interesting alternative to a direct radiating array is a reflector antenna in combination with a digital feed array as illustrated schematically in Figure 2 on the right. This architecture, which may be of special interest for low frequency radar systems operating in L- or P-band, combines the capabilities of digital beamforming with the concentrating efficiency of a large reflector antenna. The reflector could be deployable as already suggested for several forthcoming radar missions. Unfurlable reflectors are now a mature technology with extensive flight heritage in space telecommunications and satellites with lightweight mesh reflectors spanning diameters of more than $20 \mathrm{~m}$ will be launched in the near future.

The reflector based architecture offers the potential to use all feed elements simultaneously for the transmission of a broad and spill-over free beam as desired for wide swath illumination. One may even use separate waveforms for the individual feed array elements for multidimensional waveform encoding, which adds the flexibility to operate the system in optimized modes (cf. Section V). The opportunity for individual transmission and recording from the feed elements has moreover the potential to measure and adaptively compensate signal deteriorations due to deformations in the reflector structure via the transmission and reception of (ground reflected) calibration pulses.

For a paraboloidal reflector with a feed array close to the focal plane, the signals from a given direction (or a small footprint as indicated by the small red ellipses in Figure 2 on the right) correspond typically to only one or a small subset of activated feed elements. This beam direction to feed array element correspondence can be used to reduce the costs and the technical implementation complexity of a digital beamforming radar. For example, if one wants to follow the radar pulse with a high gain beam as it travels on the ground, one would not need to implement the aforementioned real-time beamsteering which involves a combination of all array elements, but it would be sufficient to simply read out the signals from different feed array elements in successive order since only one or a small number of feed elements is activated above the noise level at
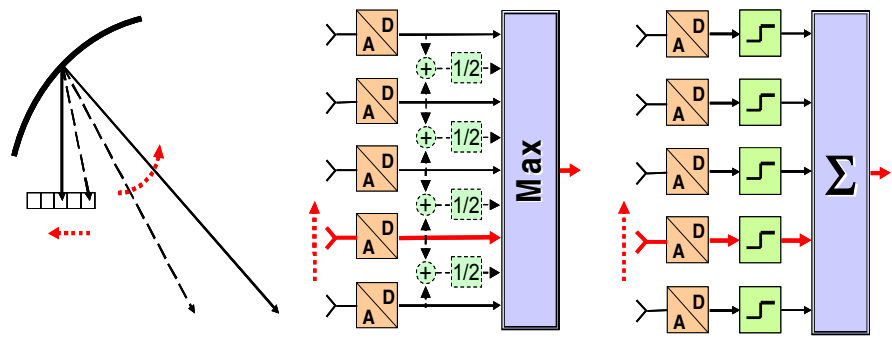

Figure 3 - The beam direction to feed element correspondence (left) enables implementations of digital beamforming with reduced complexity, e.g. via maximum selection (middle) or summation/routing of thresholded element signals (right). 


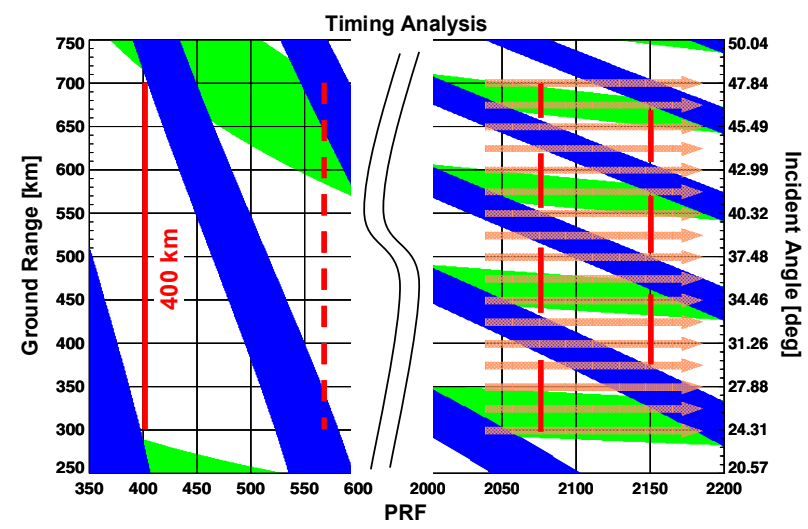

Figure $4-$ Timing for $h_{\text {sat }}=750 \mathrm{~km}$ and a duty cycle of $16 \%$.

each instant of time ${ }^{1}$. The selection, which suppresses the noise from unused Rx-modules to significantly improve the SNR, can be computed either a priori from geometric considerations or one may use appropriate thresholds to select a sparse subset and avoid the storage of irrelevant data as illustrated in the simplified sketches on the right hand side of Figure 3 (see also [15]). This results in a less complex and more cost-effective implementation of a "digital beamforming SAR" which does not necessarily need (but would nevertheless benefit from) a sophisticated on-board multi-channel real-time processor.

\section{Operational Modes}

In the previous sections we discussed different multi-channel architectures for future high-resolution wide-swath SAR systems. In the following, we introduce and compare different SAR imaging modes that take advantage from the new digital radar architectures. As a design example we consider a system which is able to map a swath width of approx. $400 \mathrm{~km}$ with an azimuth resolution of $5 \mathrm{~m}$. Such a system exceeds by far the capabilities of current spaceborne SAR sensors. To avoid a too strong variation of the incident angles we assume an orbital altitude of $750 \mathrm{~km}$.

\section{A. Multi-Channel Stripmap Mode}

We first consider a multi-aperture mapping in standard stripmap mode. The timing diagram in Figure 4 reveals that the imaging of a contiguous $400 \mathrm{~km}$ swath requires a PRF in the order

$400 \mathrm{~Hz}$. The minimum and maximum incident angles are $24^{\circ}$ and $48^{\circ}$, respectively. Assuming "full resolution" SAR imaging, the necessary antenna length to avoid azimuth ambiguities can be approximated by $l_{\text {ant }} \approx 2 v / P R F$ which yields an antenna length of $l_{\text {ant }} \approx 35 \mathrm{~m}$. The achievable azimuth resolution is then in the order of $\Delta a z \approx 20 \mathrm{~m}$. By illuminating a wider Doppler spectrum and dividing the receiver antenna into multiple azimuth apertures with individual receiver channels it becomes possible to improve the azimuth resolution to $\Delta a z \approx l_{a n t} /\left(2 N_{a z}\right)$ where $N_{a z}$ is the number of independent azimuth channels. An azimuth resolution of $5 \mathrm{~m}$ would hence require at least $N_{a z}=4$

\footnotetext{
${ }^{1}$ The number of activated feed elements depends on their distance from the focal point, i.e. more elements are activated as the scan-angle increases relative to the main beam axis. For long Tx chirp pulses, the positions of the activated elements further depend on the RF frequency, which may require either a frequency dependent selection or a summation among multiple feeds.
}
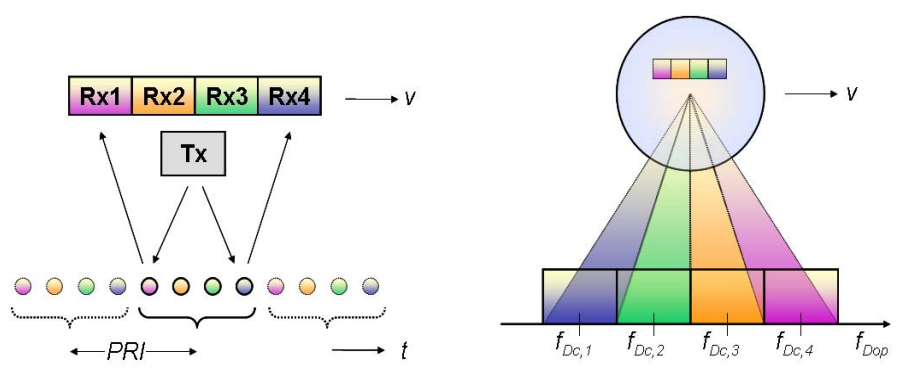

Figure 5 - Left: Multi-aperture receiver antenna for the acquisition of additional samples along the synthetic aperture. Right: Reflector antenna configuration with displaced feed elements for the simultaneous acquisition of multiple azimuth frequency sub-bands with different Doppler centroids.

channels. The resolution improvement without a rise of ambiguities is possible for both the direct radiating array and the reflector configuration. The performance gain can be understood in the former case as the acquisition of additional samples along the synthetic aperture and in the latter case as the acquisition of additional frequency sub-bands with different Doppler centroids to increase the overall azimuth bandwidth (cf. Figure 5).

\section{B. Multi-Channel ScanSAR Mode}

The main drawback of the multi-channel stripmap mode is the long antenna required for wide swath imaging. An alternative to map the $400 \mathrm{~km}$ swath is the ScanSAR (or TOPS) mode. Assuming an antenna length of $10 \mathrm{~m}$, the minimum PRF is in the order of $1.5 \mathrm{kHz}$. Timing considerations reveal that at least 6 bursts would be required to cover a $400 \mathrm{~km}$ swath. The azimuth resolution is then $\Delta a z>\left(N_{\text {burst }}+1\right) \cdot l_{\text {ant }} / 2$ where $N_{\text {burst }}$ is the number of bursts. The achievable azimuth resolution is (under optimistic assumptions) in the order of $\Delta a z \approx 35-40 \mathrm{~m}$. To achieve an azimuth resolution of $\Delta a z=5 \mathrm{~m}$ one may again employ multiple azimuth channels. The minimum number of azimuth channels is $N_{a z}>l_{\text {ant }}\left(N_{\text {burst }}+1\right) /(2 \Delta a z)$. Hence, at least 8 channels will be required. Problems arise for the multiaperture processing from the large squint angles, the varying target Doppler spectra, and the wide range of PRFs [14].

\section{Multiple Beam ScanSAR Mode}

The previous section revealed that the mapping of a wide image swath with a reasonable antenna length requires a large number of bursts. This leads to conflicts regarding performance and achievable azimuth resolution. Such problems can be mitigated by a simultaneous mapping of multiple swaths during each burst as illustrated in Figure 6. The right hand side of Figure 4 shows that it is sufficient to use only two bursts with slightly different PRFs to map an ultra-wide swath if it is possible to suppress range ambiguities and nadir echoes by digital beamforming on receive. The use of a reflector antenna based digital beamforming receiver has the possible advantage to avoid saturation effects in the relevant receiver channels that could arise in case of very strong nadir returns. The strength of the nadir signal can be further reduced by a careful design of the Tx antenna pattern and an additional improvement is possible by using different waveforms for each transmitted pulse which enables a spread of the nadir energy in the received echoes.

The two PRFs in Figure 4 on the right are sufficient to avoid azimuth ambiguities for a $7.5 \mathrm{~m}$ antenna length. In combination 


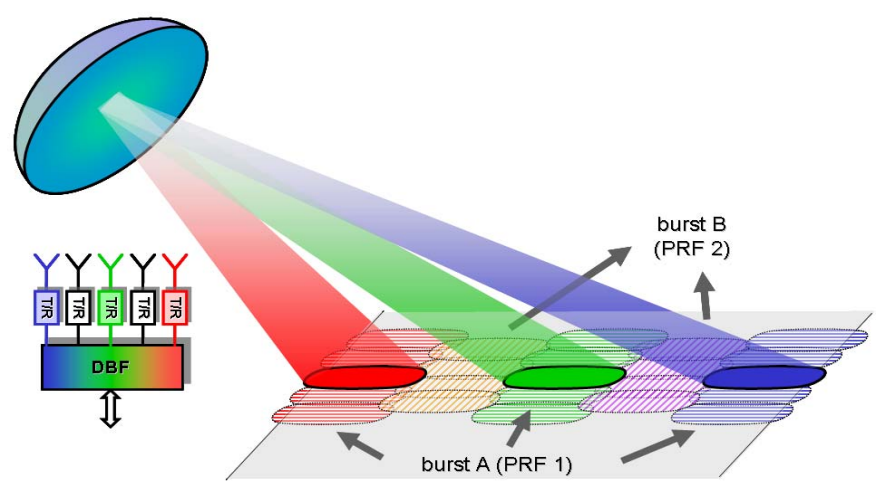

Figure 6 - Multiple-beam SAR imaging in burst mode operation

with 3 azimuth channels one obtains an azimuth resolution of $\Delta a z \approx 4 \mathrm{~m}$. The minimum antenna heights to separate the pulses from the different swaths are $0.6 \mathrm{~m}, 1.1 \mathrm{~m}$, and $4.4 \mathrm{~m}$ for X-, C-, and L-band.

An inherent peculiarity of the multiple swath ScanSAR mode is that it is not possible to adapt the burst-length to the varying ranges in order to achieve a constant azimuth resolution that is independent of slant range. In our example, the azimuth resolution varies by a factor of 1.3 across the $400 \mathrm{~km}$ swath with the best resolution in near range. On the other hand, one can combine more bursts in the far range beams to improve the radiometric resolution. If one wants to maximize the radiometric resolution for each range, one could even consider a continuous variation of the PRF during the target exposure time. As an example, a periodic linear increase of the PRF from 2040 $\mathrm{Hz}$ to $2180 \mathrm{~Hz}$ shifts the blind ranges smoothly across the swath (cf. arrows in Figure 4 on the right). For each range one obtains then a contiguous burst of Rx pulses ${ }^{2}$. The SAR focusing of each burst requires an appropriate preprocessing which interpolates the (multi-channel) azimuth raw data to a uniform sampling interval. Another opportunity arises in case of very short transmit pulses [17]. Since one looses for each range only a short segment of the synthetic aperture one may even consider a reconstruction of the complete azimuth spectrum which would allow for a range independent azimuth resolution of approx. $4 \mathrm{~m}$ with a single azimuth channel.

\section{Multidimensional WAVEFORM ENCODING}

An advanced mode to operate a multi-channel SAR is the use of spatiotemporally non-separable waveforms for each transmitted radar pulse [15]. Such waveforms are characterized by the inequality

$$
w\left(t, \theta_{e l}, \theta_{a z}\right) \neq h(t) \cdot a\left(\theta_{e l}\right) \cdot b\left(\theta_{a z}\right)
$$

where $h(t)$ describes the temporal modulation of the transmitted radar pulse, $a\left(\theta_{e l}\right)$ the weighting from the antenna pattern in elevation, and $b\left(\theta_{a z}\right)$ the weighting from the antenna pattern in azimuth. A simple example for a non-separable waveform encoding in space and time is a mere switching between different antenna beams and/or sub-aperture elements during each transmitted pulse. The beam switching in a direct radiating array can e.g. be implemented via a switching between different

\footnotetext{
${ }^{2}$ Note that the periodic jump from the highest to the lowest PRF may cause an additional short gap, which can, however, be interpolated without a notable performance decrease.
}

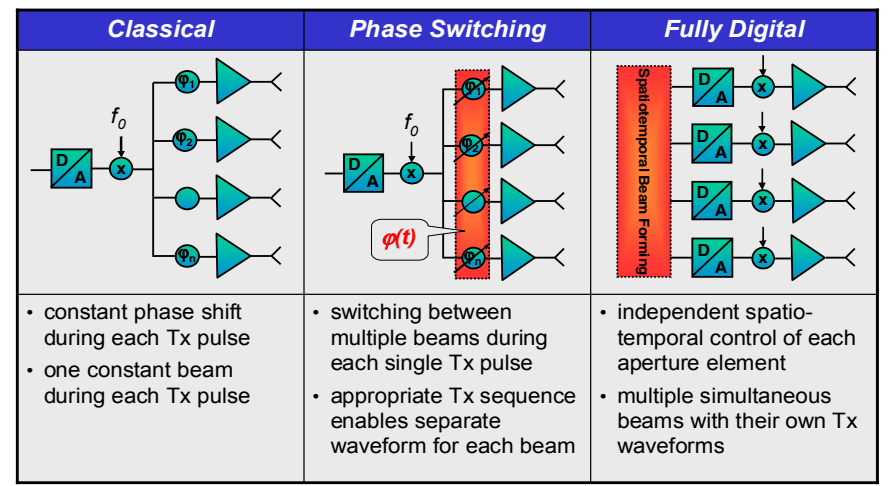

Figure 7 - Beamforming on Transmit

phase coefficients (cf. Figure 7, middle). This allows a staggered illumination of a large area during each pulse, thereby supporting a systematic distribution of the available signal energy within this area. As discussed in [15], intra-pulse beamsteering is moreover well suited to increase the transmit duty cycle without restraining the swath width. This reduces both the peak-power requirements and the recorded data volume without sophisticated on-board multi-channel beamforming. Another opportunity is the use of sub-pulses with different polarisations. If we transmit e.g. first a sub-pulse with vertical and then a sub-pulse with horizontal polarization and separate the two pulse echoes by digital beamforming on receive in elevation, we will be able to implement a fully polarimetric SAR system without the necessity to increase the PRF (cf. Figure 8). Such a mode could be of special interest for systems with a rather high receiver antenna like in the circular reflector concept.

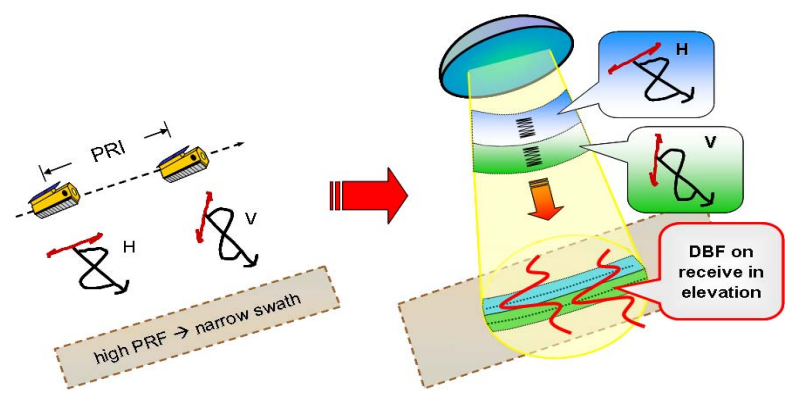

Figure 8 - Multidimensional waveform encoding for fully polarimetric operation. Left: conventional concept which requires a higher PRF and leads to a narrower swath. Right: DBF concept without change on the PRF and swath width.

The use of waveform diversity in the radar transmitter can also increase the information about the direction of a given scatterer. As a first example we consider a direct radiating array where each aperture transmits its own orthogonal waveform (cf. Figure 7 , right). The orthogonality enables a separation of the radar echoes from the different transmit signals and the spatial diversity of the transmit phase centers causes relative phase shifts between the received waveforms for a given scatterer on the ground. This additional information can then be used to suppress ambiguous returns from point like targets or to increase the sensitivity to object movements by evaluating systematic phase differences between the orthogonal radar echoes.

The performance gain from such a multidimensional 
waveform encoding can also be understood by considering the additional effective phase centre positions resulting from a multi-aperture $\mathrm{Tx} / \mathrm{Rx}$ system. Figure 9 compares the effective phase centre positions of the HRWS system (left) with the multidimensional waveform encoding technique (right). For the HRWS system, which combines a single fixed illuminator with a multi-channel receiver, one obtains for each transmitted pulse in total $N_{R x}$ effective phase centers. Their positions are spatially separated by a distance of $d_{a n t} / 2$ where $d_{a n t}$ is the distance between the $\mathrm{Rx}$ apertures in the along-track direction. The maximum distance of the phase centers is then given by $d_{\max }=d_{a n t} \cdot\left(N_{R x}-1\right) / 2$ where $N_{R x}$ is the number of channels in azimuth. The use of multidimensional waveform encoding leads now to additional phase centers, since we have to consider each $\mathrm{Tx} / \mathrm{Rx}$ aperture pair (cf. Figure 9, right). If we assume the same number $N=N_{R x}=N_{T x}$ and equal positions for the Tx and $\mathrm{Rx}$ apertures, we obtain in total $2 N-1$ independent phase centre positions which span a total length of $d_{\max }=(N-1) \cdot d_{\text {ant }}$. This length is twice the length of the classical DPCA system employing a single transmitter. The additional phase centers provide hence an increased number of azimuth samples along the synthetic aperture with the potential for improved ambiguity suppression. One may hence reduce either the PRF or the overall antenna length by a factor of two. Another opportunity is an enhanced detection and parameter estimation performance in a multi-baseline along-track interferometer and/or ground moving target indication (GMTI) system due to the increased length of the total along-track baseline.
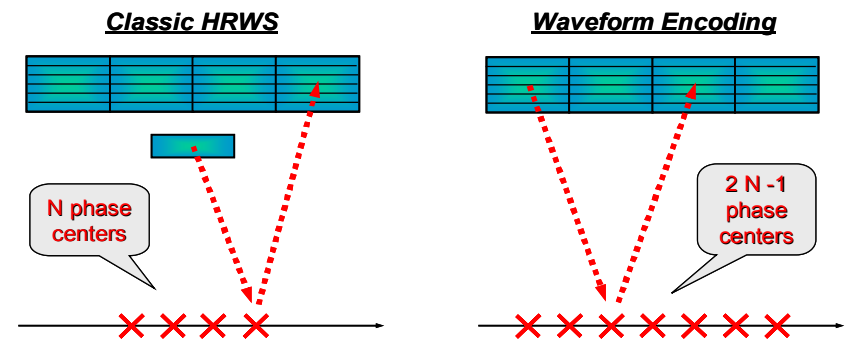

Figure 9 - Additional phase centers by multidimensional waveform encoding

On a first sight, one might believe that orthogonal $\mathrm{Tx}$ waveforms are also well suited to reduce azimuth ambiguities in a high-resolution wide-swath SAR imaging system. However, the mere use of simultaneously transmitted orthogonal waveforms will only disperse - but not suppress - the ambiguous energy, thereby making this approach only suitable for the attenuation of ambiguous returns from point-like targets in specialized scenarios. A full suppression of ambiguous returns from distributed targets can, however, be obtained by combining the spatial transmit diversity in azimuth with digital beamforming on receive in elevation. For this, the signals from the azimuth apertures are not transmitted simultaneously but in sequence by dividing the total Tx pulse again into multiple subpulses where the number of sub-pulses corresponds to the number of azimuth apertures. The scattered signals from the different sub-pulses will then -at each instant of time- arrive from different elevation angles and it becomes possible to separate the radar echoes from the different sub-pulses by digital beamforming on receive in elevation. This spatial filtering will hence suppress, and not only disperse, the ambiguous energy from distributed scatterers.
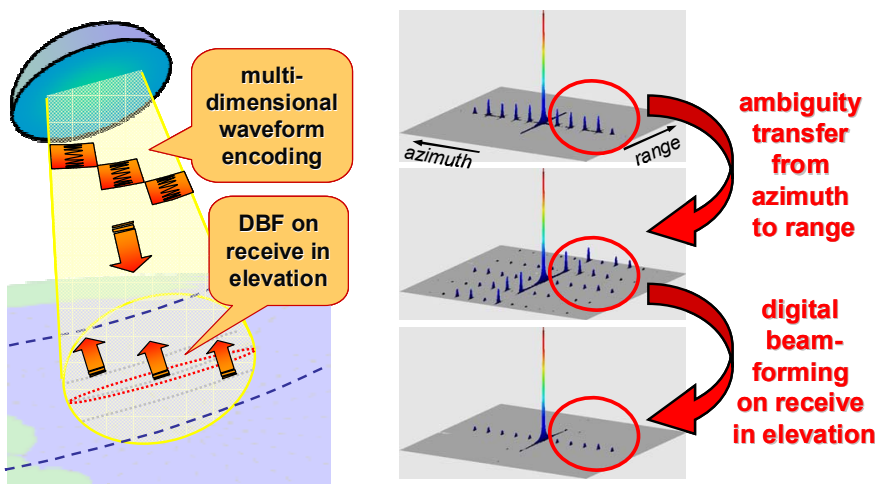

Figure 10 - Multidimensional waveform encoding in azimuth

An alternative to the sequential transmission from multiple azimuth apertures is the formation of multiple narrow azimuth beams in the transmitter. A sequence of full-bandwidth chirp signals is then transmitted while switching between different azimuth beams from sub-pulse to sub-pulse, as illustrated in Figure 10 on the left. This specific illumination sequence results for each point on the ground in multiple and mutually delayed chirp signal returns. If we consider now a scatterer at a given range, one will at each instance of time only receive the scattered signal from one sub-pulse while the other sub-pulses lead to a superposition of the received signal with range ambiguous echoes from scatterers located at different ranges. These different ranges are in turn associated with different look angles in elevation. It is hence possible to suppress the ambiguous returns from different ranges by digital beamforming on receive in elevation which enables a clear and unambiguous separation of the received echoes from the different azimuth beams (cf. Figure 10, right). The echoes from multiple azimuth beams are finally combined coherently to recover the full Doppler spectrum for high azimuth resolution. This combination is equivalent to a signal reconstruction from a multi-channel bandpass decomposition, where the individual bandpass signals correspond to narrow band azimuth spectra with different Doppler centroids. A detailed description and the corresponding processing algorithms can be found in [15].

\section{CONCLUSIONS}

We have introduced several new SAR system concepts for the acquisition of high resolution radar images with very wide swath coverage. These concepts rely on the combination of advanced multi-channel SAR front-end architectures with novel operational modes. The architectures differ regarding their complexity and it has been shown that even a low number of channels is already well suited to significantly improve the performance and to overcome some fundamental limitations inherent to classical SAR systems. The more advanced multichannel concepts will in the future greatly benefit from recent developments in integrated microwaves and semi-conductor technologies [17]. One opportunity is the systematic combination of spatiotemporal radar waveform encoding on transmit with multi-aperture digital beamforming on receive which enables several new and powerful SAR imaging modes for a wide range of remote sensing applications. Examples are superior ambiguity suppression, improved geometric resolution and radiometric sensitivity as well as the availability of 
additional phase centres for along-track interferometry and moving object indication.

Digital beamforming on transmit allows furthermore a flexible distribution of the RF signal energy on the ground. This enables not only a switching between different SAR modes like Spotlight, ScanSAR and HRWS stripmap, but it allows also for the simultaneous combination of multiple imaging modes in one and the same data acquisition. An example for such an interleaved operation is a spotlight imaging of an area of high interest in combination with a simultaneous wide swath SAR mapping for interferometric applications. This can be achieved by enhancing the multidimensional waveform encoding with additional sub-pulses that steer highly directive transmit beams to some specific areas on the ground as illustrated in Figure 11 on the left. By this, one obtains a high Tx gain and a longer illumination time along the synthetic aperture, which will improve both the radiometric and the geometric resolution for the localized areas of high interest. Such a hybrid mode is well suited to satisfy otherwise contradicting user requirements like the conflict between a continuous interferometric background mission and a high-resolution imaging request.

The selection of the spatiotemporal excitation coefficients for the individual $\mathrm{Tx}$ apertures could even be made adaptive by evaluating the recorded samples from previous signal returns. By this, a closed loop will be formed between the radar sensor and its environment, which allows for a maximization of the information that can be derived about the imaged scene for a given RF power budget (cf. Figure 11, right). In analogy to the information theoretic modeling of multiple-input multipleoutput (MIMO) communication systems, such an optimization could then be regarded as maximizing the mutual information between the recorded radar signals and the scatterer distribution on the ground, thereby making optimum use of the channel capacity provided by the multiple antenna $\mathrm{Tx} / \mathrm{Rx}$ radar system. For illustration, one may consider the simple case of an automatic compensation of angular variations in the received $\mathrm{Rx}$ power being caused by, e.g., range differences, inhomogeneous atmospheric RF signal attenuation, and/or spatial variations in the first-order scattering statistics of the imaged scene. Another opportunity is the use of longer azimuth illumination times and higher range bandwidths for selected regions with high contrast and/or fast changes. Such features could be indicators for areas of high interest, therefore calling also for a higher spatial and/or radiometric resolution. Such a system can also be regarded as a first step towards a cognitive radar which directs its resources to areas of high interest in analogy to the selective attention mechanisms of the human visual system with its saccadic eye movements [18]. The rising interest in such systems is also well documented in recent literature [19].

The full exploitation of all opportunities arising from such an adaptive multidimensional waveform encoding requires of course new SAR system design and optimization strategies. For example, the derivation of optimized waveforms may incorporate elements from Shannon's information theory. This will not only help to maximize the information content derived from the imaged scene, but it is also well suited to eliminate redundancies in the multi-channel SAR data, thereby alleviating possible downlink bottlenecks.

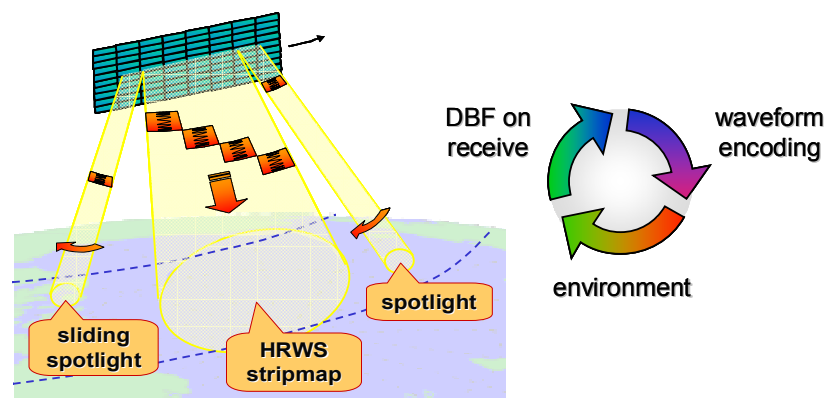

Figure 11 - Hybrid and adaptive SAR imaging modes

\section{REFERENCES}

[1] J. C. Curlander, R. N. McDonough, Synthetic Aperture Radar: Systems and Signal Processing. New York: Jon Wiley \& Sons, 1991.

[2] K. Tomiyasu, "Conceptual performance of a satellite borne, wide swath synthetic aperture radar," IEEE Trans. Geosc. Remote Sensing, vol. 19, pp. 108-116, 1981.

[3] A. Jain, "Multibeam synthetic aperture radar for global oceanography," IEEE Trans. Antennas and Propagation, vol. 27, pp. 535-538, 1979.

[4] W. Carrara, R. Goodman, R. Majewski, Spotlight Synthetic Aperture Radar: Signal Processing Algorithms. Boston: Artech House, 1995.

[5] A. Currie, M. A. Brown, "Wide-swath SAR," IEE Proceedings F - Radar and Signal Processing, vol. 139, pp. 122-135, 1992.

[6] J. P. Claassen, J. Eckerman, "A system for wide swath constant incident angle coverage," in Proc. Synthetic Aperture Radar Technology Conference, Las Cruces, New Mexico, USA, 1978.

[7] B. R. Jean, J. W. Rouse, "A multiple beam synthetic aperture radar design concept for geoscience applications," IEEE Transactions on Geoscience and Remote Sensing, vol. 21, pp. 201-207, 1983.

[8] H. Griffiths, P. Mancini, "Ambiguity suppression in SARs using adaptive array techniques," in Proc. IGARSS, Espoo, Finland, pp. 1015$1018,1991$.

[9] G. D. Callaghan, I. D. Longstaff, "Wide-swath space-borne SAR using a quad-element array," IEE Proc. Radar Sonar Navig., vol. 146, pp. 159$165,1999$.

[10] M. Suess, B. Grafmueller, R. Zahn, "A novel high resolution, wide swath SAR system," in Proc. IGARSS, Sydney, Australia, pp. 1013$1015,2001$.

[11] G. Krieger, N. Gebert, A. Moreira, "Unambiguous SAR signal reconstruction from nonuniform displaced phase center sampling," IEEE Geoscience and Remote Sensing Letters, vol. 1, pp. 260-264, 2004.

[12] C. Fischer, C. Heer, G. Krieger, and R. Werninghaus, "A high resolution wide swath SAR," in Proc. EUSAR, Dresden, Germany, 2006.

[13] N. Gebert, G. Krieger, A. Moreira, "Digital Beamforming on Receive: Techniques and Optimization Strategies for High Performance SAR Imaging," IEEE Trans. Aerospace Science and Technology, in print.

[14] N. Gebert, G. Krieger, A. Moreira, "Multi-channel ScanSAR for highresolution ultra-wide-swath imaging", EUSAR 2008.

[15] G. Krieger, N. Gebert, A. Moreira, "Multidimensional Waveform Encoding: A New Digital Beamforming Technique for Synthetic Aperture Radar Remote Sensing," IEEE Trans. Geoscience and Remote Sensing, vol. 46, no.1, pp. 31-46, 2008.

[16] G. Krieger, A. Moreira, "Spaceborne Bi- and Multistatic SAR: Potential and Challenges," IEE Proceedings - Radar, Sonar and Navigation, vol. 153, no. 3, pp. 184-198, 2006.

[17] M. Ludwig, M. Suess, N. Le Gallou, "Technologies for advanced SAR systems", EUSAR'06, Dresden, Germany, 2006.

[18] K. Schill, E. Umkehrer, S. Beinlich, G. Krieger, and C. Zetzsche, "Knowledge-based scene analysis with saccadic eye-movements," Journal of Electronic Imaging, vol. 10, pp. 152-160, 2001.

[19] F. Gini, "Knowledge-Based Systems for Adaptive Radar [Guest editorial]," IEEE Signal Processing Magazine, vol. 23, pp. 14-17, 2006. 